Gut, 1975, 16, 509-513

\title{
Gastric emptying following vagotomy and antrectomy and proximal gastric vagotomy
}

\author{
H. KALBASI, F. R. HUDSON, A. HERRING, S. MOSS, H. I. GLASS, AND \\ J. SPENCER
}

From the Departments of Surgery and Medical Physics, Royal Postgraduate Medical School, Hammersmith Hospital, London

SUMMARY Gastric emptying of solid meals labelled with ${ }^{129} \mathrm{Cs}$ was studied in patients for up to one year after vagotomy and antrectomy or after proximal gastric vagotomy. Significant delay was found one month after vagotomy and antrectomy but this had returned to normal by six months. No delay was found after proximal gastric vagotomy.

The effect of posture on gastric emptying was also studied in the same subjects. No significant differences were found between gastric emptying in the supine or sitting positions after solid meals.

The technique of monitoring clearance from the stomach of a meal containing a radioactive tracer has been widely used in recent years in patients with a variety of gastric disorders (Jones, Clark, Kocak, Cox, and Glass, 1970; Tinker, Kocak, Jones, Glass, and Cox, 1970; Colmer, Owen, and Shields, 1973; and van Dam, 1974). Early workers used external counters but the use of computerized gamma-camera systems is becoing more common (Cowley, Vernon, Jones, Glass, and Cox, 1972; Hancock, BowenJones, Dixon, Dymock, and Cowley, 1974).

The recent study of van Dam (1974) indicates that the range of emptying rates is large in a group of normal subjects but that individuals appear to have characteristic and repeatable values. However, the effects on gastric emptying rates of a number of important variables remain to be fully resolved and there is no standard technique for the acquisition and presentation of data.

This paper presents the results of a comparative study of the effects of truncal vagotomy and antrectomy and of proximal gastric vagotomy (Amdrup and Jensen, 1970; Johnston and Wilkinson, 1970) on gastric emptying. Emptying patterns have been investigated by comparison of measurements performed during the period from one month to one year following operation.

Among the factors which may affect the results of such measurements are the character of the meal, the posture of the subject, the length of time of the observations, and the mode of presentation of the

Received for publication 17 April 1975. results. In the present study a standard physiological solid meal was used. The possible effect of posture on the identification of gastric incontinence was investigated in a parallel study in which patients were studied in both the supine and sitting positions. The results are compared with those obtained for a control group who had not undergone gastric surgery.

\section{Patients and Methods}

Gastric emptying measurements were performed in a total of 19 patients who had undergone proximal gastric vagotomy and 16 patients who had undergone truncal vagotomy and antrectomy for duodenal ulcer. The operations were randomly allocated as part of a controlled clinical trial. Studies were performed at one month after operation with eight patients in each group, at four months with nine patients in each group, at six months with 16 truncal and vagotomy and 11 proximal gastric vagotomy patients, and at 12 months with seven truncal vagotomy and antrectomy and five proximal gastric vagotomy patients.

Postural studies were performed in eight similar subjects after proximal gastric vagotomy and seven after truncal vagotomy and antrectomy. Controls for both studies were provided by tests in seven healthy volunteers.

The technique was as outlined by Cowley et al (1972). The subjects fasted overnight and were given a meal of two scrambled eggs, two slices of buttered bread, a spoonful of jam and $300 \mathrm{ml}$ of 
milk. The milk was mixed with $100 \mu \mathrm{Ci}$ of ${ }^{129} \mathrm{Cs}$, absorbed on to zirconium phosphate resin, to provide a solid, inert carrier. The ${ }^{129} \mathrm{Cs}$, which has a half-life of 32 hours and emits $\gamma$ rays of $375 \mathrm{KeV}$ $(48 \%)$ and $416 \mathrm{KeV}(25 \%)$ together with a number of weaker $\gamma$ rays, was chosen because of its acceptable dosimetry characteristics together with its convenience for preparation in a form which is not absorbed from the stomach nor released from the carrier resin in the presence of strong acid. The radiation dose to the lower large intestine (the critical organ) is 3.7 mrads per $\mu \mathrm{Ci}$ of ${ }^{129} \mathrm{Cs}$ administered.

On completion of the meal the patients were positioned under the gamma camera and collection of data was started. This was normally within five minutes of the end of the meal. Data were stored in digital form on magnetic tape and a time function showing the rate of decrease of counts in the stomach area was computed. The selection of the area was based on the distribution of activity in the first four minutes of acquisition of data, and recording was continued for 90 minutes. 'Second posture' studies were normally carried out within two weeks of the first study. 'Sitting' studies were performed with the subject seated on a couch with the trunk at an angle of $45^{\circ}$ from the horizontal. Although not a natural upright posture, this was the best compromise compatible with the use of the gamma camera.

Statistical comparisons of the groups were based on a two-tailed Wilcoxon ranking test. Analysis of the postural data was made with a paired test; all other tests were unpaired.

\section{Results}

The results of the investigation are shown in figs 1 and 2 in the form of estimated stomach contents at four selected times through the 90 -minute study as a percentage of the initial contents, each value being the average for a five-minute period.

The changes in emptying patterns in the 19 proximal gastric vagotomy and 16 vagotomy and antrectomy patients studied at one, four, six, or 12 months after operation are compared with the results for a control group in figure 1 .

One month after operation patients who had undergone vagotomy and antrectomy had a significantly slower emptying than the control group (P $<0.01)$. After four months this was returning towards normal and by six months no difference between this group and the controls could be observed. The proximal gastric vagotomy patients at no stage showed significant differences from the controls. These findings correlate well with clinical

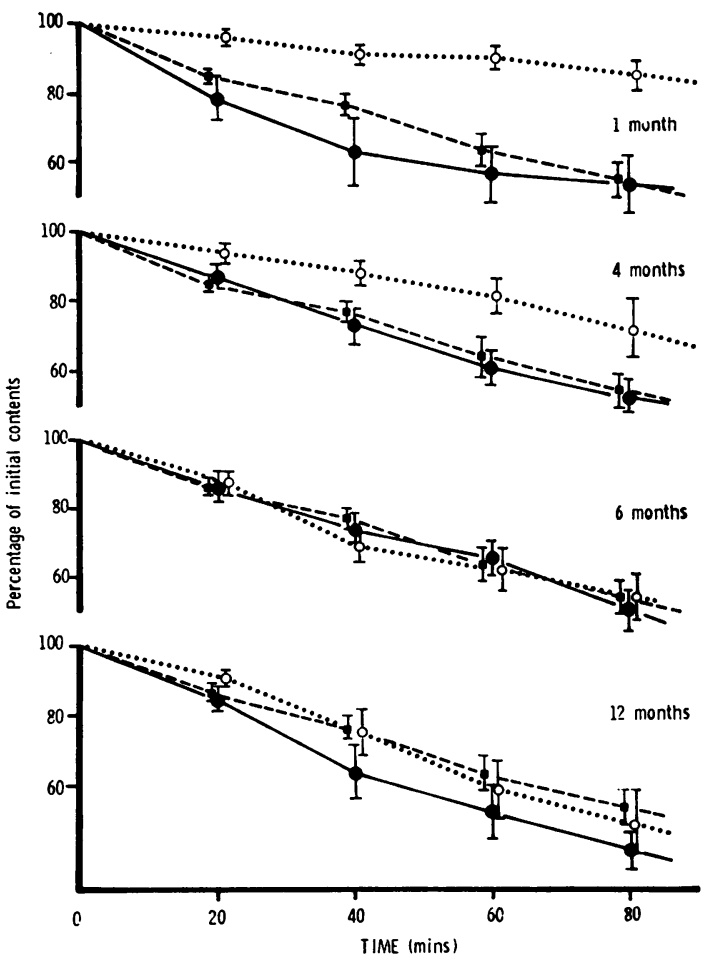

Fig 1 Gastric emptying patterns at different periods after vagotomy and antrectomy $(O)$, after proximal gastric vagotomy (O), and in a control group ( $(\square)$ (single study, no operation) (mean $\pm S E M)$.
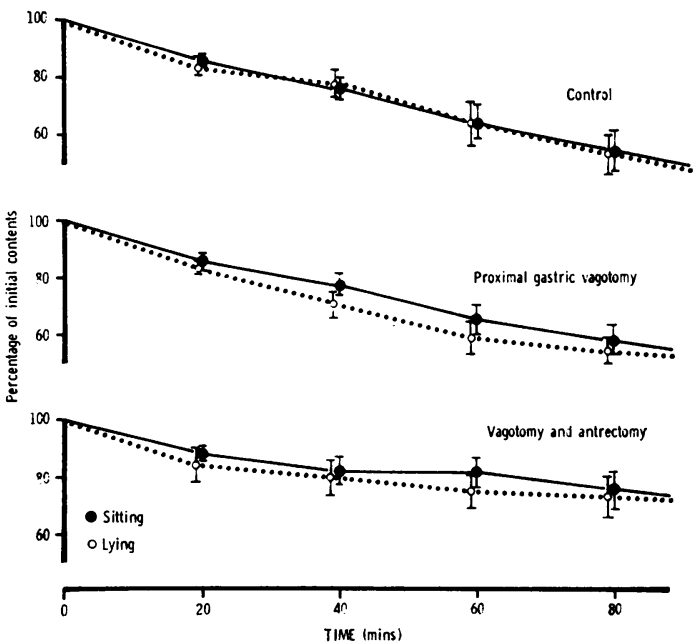

Fig 2 Gastric emptying patterns observed in the supine $(O)$ and in the sitting $(\bullet)$ positions in a control group (no operation) and after proximal gastric vagotomy and after vagotomy and antrectomy $($ mean $\pm S E M)$. 
assessment one month after operation when 10 patients after antrectomy had moderate to severe symptoms of epigastric fullness, whereas this symptom was present in only five after proximal vagotomy, this difference being statistically significant $\left(\chi^{2}=4 \cdot 23, \mathrm{P}<0.05\right)$. These symptoms abated later, which accords well with the observed improvement in gastric emptying.

Postural comparison (fig 2) were made in seven patients after vagotomy and antrectomy, in eight after proximal gastric vagotomy, and in seven controls. No significant difference was found between gastric emptying rates in the two positions in any of the groups. However, comparisons between the groups do show the vagotomy and antrectomy patients to have cleared significantly less of the meal $(P<0.01)$ than the control group at the end of the study. No significant difference was found between the proximal gastric vagotomy and control groups.

\section{Discussion}

ANALYSIS

One problem which arises in the analysis of the gastric emptying curves is the choice of the method used to present the data. Many previous workers have used a half-emptying time ( $\left.\mathrm{T}_{2} \frac{1}{2}\right)$, making the assumption that the emptying curve can be described by a monoexponential function. While this is frequently valid, in a substantial minority of studies the monoexponential assumption is a very poor approximation, as Hancock et al (1974) have commented. The data for one subject in the present study showing several distinct phases of emptying are shown in figure 3 . This truncal vagotomy and

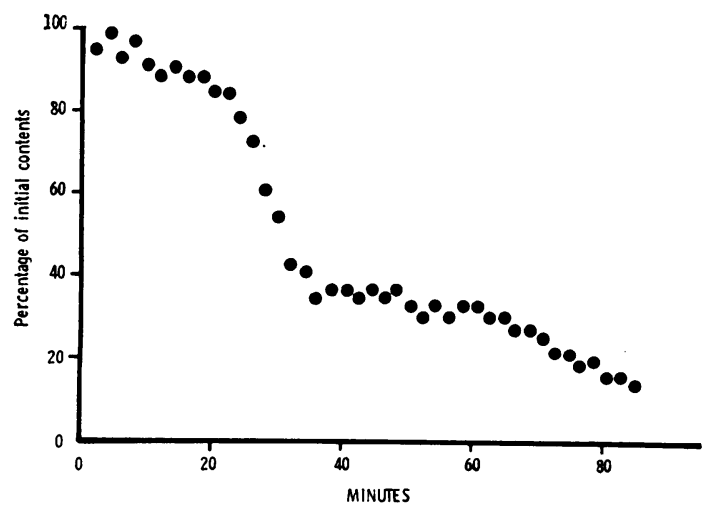

Fig 3 Example of gastric emptying pattern in a single individual who showed several clearly defined emptying phases after vagotomy and antrectomy. antrectomy patient showed a very similar emptying pattern in two studies six months apart. Emptying curves of this type were found in some $20 \%$ of studies.

An additional problem to be considered is that frequently the period of measurement is significantly less than the assigned $T \frac{1}{2}$. This may lead to large errors due to failure of the monoexponential approximation.

The present results are shown in the form of the percentage of the meal remaining in the stomach at equally spaced time intervals, as this retains more of the information in the emptying curve and permits comparison of early or late emptying patterns. The disadvantage of this method of analysis is that all readings are biased by the initial reading.

For comparison the table shows the relation between a range of typical half-times and the percentage contents at different intervals which they would represent.

\begin{tabular}{rllll}
\hline$T \frac{1}{2}$ & \multicolumn{2}{l}{$T$ (minutes) } & \\
\cline { 2 - 5 } & 20 & 40 & 60 & 80 \\
\hline 60 & 80 & 63 & 50 & 40 \\
100 & 86 & 76 & 66 & 57 \\
200 & 94 & 87 & 81 & 76 \\
\hline
\end{tabular}

Table Stomach contents at $T=20,40,60$ and 80 minutes as a percentage of $T=0$ contents corresponding to monoexponential emptying with $T \frac{1}{2}=60,100$, and 200 minutes

COMPARISON OF TRUNCAL VAGOTOMY AND ANTRECTOMY WITH PROXIMAL GASTRIC VAGOTOMY

Dozois, Kelly, and Code (1971) studied the effect of distal antrectomy on the gastric emptying of liquids and solids (plastic spheres) in dogs. They found that distal antrectomy hastened liquid empyting slightly although the square root of the volume of instillate remaining still declined linearly with time. In contrast the antrectomy markedly increased the gastric emptying of spheres. They concluded that the distal antrum and pylorus have a minor role in the gastric emptying of liquids but are important to the emptying of solids.

Wilkinson and Johnston in 1973 studied the emptying of a semi-solid-food-barium meal in patients following selective vagotomy and drainage, truncal vagotomy and drainage, and highly selective vagotomy without drainage. They concluded that gastric emptying and small bowel transit times were closer to normal in patients who had undergone highly selective vagotomy (proximal gastric vagotomy) than in patients who had undergone 
truncal or selective vagotomy with pyloroplasty. They found no evidence of delayed gastric emptying after highly selective vagotomy.

The effect of preserving antral innervation and of a pyloroplasty on gastric emptying after vagotomy in man was studied by Clarke and Alexander-Williams in 1973. Their patients had one of four operations (selective vagotomy, proximal gastric vagotomy, selective vagotomy plus pyloroplasty or proximal gastric vagotomy plus pyloroplasty). Gastric emptying of a hypertonic liquid meal was assessed before and three to four months after operation. They concluded that selective vagotomy without a drainage procedure results in gastric retention. Proximal gastric vagotomy without a drainage procedure was not found to lead to gastric retention and initial gastric emptying is more rapid following proximal gastric vagotomy but the final emptying time is the same as before operation. This operation was found to have the least effect on the pattern of gastric emptying. They found that pyloroplasty added to either selective or to proximal gastric vagotomy results in a loss of the normal regulation of gastric emptying, very rapid initial gastric emptying, and a significant increase in the incidence of dumping.

The present results show gross delay in gastric emptying at one month after vagotomy and antrectomy which had returned towards normal at four months. At one year emptying was within the normal range in this group. No significant difference from normal gastric emptying was found at any period after proximal gastric vagotomy.

Our results cannot be compared directly with those of Dozois et al (1971) or with those of Clarke and Alexander-Williams (1973) as we have used a physiological solid meal. Wilkinson and Johnston (1973), who used a semi-solid barium food, found results similar to ours following proximal gastric vagotomy. Our results for truncal vagotomy and antrectomy are similar to those obtained after vagotomy and pyloroplasty by Cowley et al (1972), who found that the gastric emptying of a solid meal was grossly delayed one to four weeks after operation. They found that emptying then returned towards normal eight weeks later.

Our findings indicate that at all periods after proximal gastric vagotomy gastric emptying is within normal limits. After truncal vagotomy and antrectomy, although the emptying is delayed initially, it returns to normal six months after operation.

POSTURAL EFFECTS

The postural effect on stomach emptying rates has been previously investigated by McKelvie (1970) who found that a small but definite increase in gastric emptying time was caused in preoperative patients by a change from a seated position to a left-sided position. He also found that in patients who had undergone a vagotomy and drainage operation the emptying time was prolonged by a factor of 3 by a similar change in position. However, McKelvie's work was based on the emptying of liquid meals, ie, water or reconstituted milk, and in postoperative patients the initial emptying times were particularly fast.

Hancock et al (1974) concluded in another study that the stomach was incontinent after both vagotomy and drainage and proximal gastric vagotomy without drainage. Ten patients were studied less than three months after vagotomy, five after truncal vagotomy and drainage, five after proximal gastric vagotomy. The gastric emptying of a meal of half a cupful of porridge, $250 \mathrm{ml}$ of milk of which half was in the porridge, and one slice of bread and marmalade labelled with ${ }^{51} \mathrm{Cr}$ was monitored with a gamma camera for 90 minutes. Tests were performed twice within five days in each subject, once with the subject lying supine and once with the subject standing. They found after both types of vagotomy that emptying rates were significantly faster when standing than when lying.

The present findings differ from those of McKelvie (1970) and Hancock et al (1974) but no direct comparison of results can be made. McKelvie's data were obtained with a liquid test meal. Hancock used a physiological solid meal but his patients were in the supine and in the standing positions while the present data were obtained for patients in the supine and sitting positions, ie, with the spine at $45^{\circ}$ to the horizontal. Hancock and his colleagues also performed their studies within three months after vagotomy while the current group of patients was studied between two weeks and one year after operation. Also the present patients had undergone antrectomy as opposed to the pyloroplasty used for Hancock's patients, though it is difficult to see how the difference in results can be explained on this basis. As the antrum may well have been an important motor function in emptying, antrectomy could conceivably delay emptying, countering the absence of a pylorus. It is difficult, however, to explain the absence of a postural effect on emptying after proximal gastric vagotomy in our subjects when compared with those of Hancock et al (1974) except in terms of the different positions used in each study. Hancock found no postural difference for normal subjects, in agreement with the current data.

\section{Conclusions}

The gastric emptying patterns of patients in the year 
following vagotomy and antrectomy or proximal gastric vagotomy were compared with those of a control group. The vagotomy and antrectomy patients showed significant delay in gastric emptying one month after operation. This delay was reduced at four months and was not apparent at later periods. The proximal gastric vagotomy patients did not show significant deviations from normal emptying patterns.

A parallel study of the effect of posture in these three groups showed no significant differences in gastric emptying which could be assigned to differences between the supine and sitting positions. It is concluded that any differences which exist are of small scale or are restricted to particular groups of patients such as those with rapid emptying patterns. It seems desirable, however, to standardize future studies on the sitting position as this is closer to normal conditions.

The authors wish to acknowledge the help and assistance which they have received from their colleagues in the Department of Surgery and of Medical Physics and in the MRC Cyclotron Unit. Particular thanks are due to Miss Harrison and the Dietetic Kitchen staff and to Miss Jenny Eckford for her secretarial help. This work was supported in part by a grant from the Medical Research Council.
References

Amdrup, E., and Jensen, H. E. (1970). Selective vagotomy of the parietal cell mass preserving innervation of the undrained antrum. Gastroenterology, 59, 522-527.

Clarke, R. J., and Alexander-Williams, J. (1973). The effect of preserving antral innervation and of a pyloroplasty on gastric emptying after vagotomy in man. Gut, 14, 300-307.

Colmer, M. R., Davies, W. T., Owen, G. M., and Shields, R. (1969). 'Dumping' after vagotomy and pyloroplasty. Brit. J. Surg., 56, 702.

Cowley, D. J., Vernon, P., Jones, T., Glass, H. I., and Cox, A. G. (1972). Gastric emptying of solid meals after truncal vagotomy and pyloroplasty in human subjects. Gut, 13, 176-181,

van Dam, A. P. M. (1974). The gamma camera in clinical evaluation of gastric emptying. Radiology, 110, 155-157.

Dozois, R. R., Kelly, K. A., and Code, C. F. (1971). Effect of distal antrectomy on gastric emptying of liquids and solids. Gastroenterology, 61, 675-681.

Hancock, B. D., Bowen-Jones, E., Dixon, R., Dymock, I. W., and Cowley, D. J. (1974). The effect of metoclopramide on gastric empyting of solid meals. Gut, 15, 462-467.

Johnston, D., and Wilkinson, A. R. (1970). Highly selective vagotomy without a drainage procedure in the treatment of duodenal ulcer. Brit. J. Surg., 57, 289-296.

Jones, T., Clark, J. C., Kocak, N., Cox, A. G., and Glass, H. I. (1970). Measurement of gastric emptying using the scintillation camera and ${ }^{120} \mathrm{Cs}$. Brit. J. Radiol., 43, 537-541.

McKelvie, S. T. D. (1970). Gastric incontinence and post vagotomy diarrhoea. Brit. J. Surg., 57, $741-747$.

Tinker, J., Kocak, N., Jones, T., Glass, H. I., and Cox, A. G. (1970). Supersensitivity and gastric emptying after vagotomy. Gut, 11, 502-505.

Wilkinson, A. R., and Johnston, D. (1973). Effect of truncal, selective and highly selective vagotomy on gastric emptying and intestinal transit of a food-barium meal in man. Ann. Surg., 178, 190-193. 